\title{
Association between enhancement patterns and parameters of contrast-enhanced ultrasound and microvessel distribution in breast cancer
}

\author{
XIAOKANG $\mathrm{LI}^{1-4^{*}}$, YAQING $\mathrm{LI}^{2-5^{*}}$, YING $\mathrm{ZHU}^{1-4}, \mathrm{LI} \mathrm{FU}^{2-5}$ and PEIFANG LIU ${ }^{1-4}$ \\ ${ }^{1}$ Department of Breast Imaging, Tianjin Medical University Cancer Institute and Hospital, \\ National Clinical Research Center for Cancer; ${ }^{2}$ Key Laboratory of Breast Cancer Prevention and Therapy, \\ Tianjin Medical University, Ministry of Education; ${ }^{3}$ Key Laboratory of Cancer Prevention and Therapy; \\ ${ }^{4}$ Tianjin's Clinical Research Center for Cancer; ${ }^{5}$ Department of Breast Cancer Pathology and Research Laboratory, \\ Tianjin Medical University Cancer Institute and Hospital, National Clinical Research Center for Cancer, \\ Tianjin 300060, P.R. China
}

Received November 4, 2016; Accepted November 20, 2017

DOI: $10.3892 / \mathrm{ol} .2018 .8078$

\begin{abstract}
The aim of the present study was to evaluate the association between enhancement patterns and parameters in contrast-enhanced ultrasonography (CEUS) and microvessel distribution and histological prognostic tumor characteristics of breast cancer. Between September 2011 and April 2013, 109 breast lesions were prospectively analyzed, which were classified into Breast Imaging Reporting and Data System class 4-5 by conventional ultrasound. Before core-needle biopsy, CEUS examination was performed. Different enhancement patterns (homogeneous, peripheral and regional enhancement) were evaluated. Following CEUS, biopsies were performed at the same body positions under guidance of ultrasound. Parameters (wash-in time, peak intensity, time to peak, area under the time-intensity curve, ascending slope) of CEUS at corresponding puncture sites were recorded. Microvessel density (MVD) and pathological prognostic factors, including histological grade, lymph node status, tumor-node-metastasis stage, estrogen and progesterone receptor status, $\mathrm{Ki}-67$ proliferative
\end{abstract}

Correspondence to: Dr Ying Zhu, Department of Breast Imaging, Tianjin Medical University Cancer Institute and Hospital, National Clinical Research Center for Cancer, 24 Huan-Hu-Xi Road, Tianjin 300060, P.R. China

E-mail: zhuying1881@msn.com

Professor Li Fu, Department of Breast Cancer Pathology and Research Laboratory, Tianjin Medical University Cancer Institute and Hospital, National Clinical Research Center for Cancer, 24 Huan-Hu-Xi Road, Tianjin 300060, P.R. China

E-mail: fuli@tijmu.edu.cn

*Contributed equally

Key words: ultrasound, contrast media, breast cancer, microvessel density, histological grade index and human epidermal growth factor receptor 2 overexpression, were determined. Mann-Whitney U test, Spearman's rank-correlation test and independent-samples t-tests were used for evaluation. Core-needle biopsy or surgical resection specimens of 99 malignant breast lesions were included in the present study. Of the 99 malignant lesions, 44 lesions exhibited homogeneous enhancement, 43 exhibited peripheral enhancement and 12 exhibited regional enhancement. Lesions with peripheral enhancement exhibited a higher peripheral/central ratio of MVD compared with lesions with homogeneous enhancement $(\mathrm{P}<0.001)$. Peak intensity and ascending slope ratios of tumor periphery/center were correlated with the corresponding MVD ratios ( $\mathrm{P}=0.017$ and $\mathrm{P}=0.016$, respectively). MVD ratio was positively correlated with histological grade $(\mathrm{P}=0.003)$. In conclusion, the enhancement patterns and parameters of CEUS may not only reflect the microvessel distribution but also indirectly indicate the histological grade of breast cancer.

\section{Introduction}

Breast cancer is a common malignancy in women. Angiogenesis serves an important role in the genesis and development of various tumors, including breast, colon, pancreatic and prostatic cancer $(1,2)$. Out of all imaging techniques, computerized tomography (CT) and magnetic resonance imaging (MRI) are able to quantify the perfusion blood volume and permeability in the level of capillaries so as to reveal the hemodynamics of tumors with good spatial and temporal resolution; however, application of these technique in breast tumor is restricted by limitations, including high radioactivity of $\mathrm{CT}$, and the complexity and time-consumption of MRI. Conventional doppler ultrasonography is not sensitive to low-velocity blood flow, which restricts its application in the assessment of neovascularization in tumor. Contrast enhanced ultrasonography (CEUS) make it possible to reflect morphologic and functional changes of microvessel perfusion in tumors. A previous study reported that CEUS may be useful for distinguishing malignant breast lesions from benign ones (3). The possibility of using CEUS for the evaluation 
of tumor perfusion following chemotherapy or other local antiangiogenic treatments has been reported (4). A previous study revealed that CEUS patterns and perfusion parameters are well correlated with the microvascular density (MVD) values and distribution in lesion sites, which have focused on the ultrasonographic characteristics of high- and low-MVD lesions (5), This study focused on the correlation between regional perfusion patterns and microvascular distributions in breast cancer. In addition, Wan et al (6) reported that certain enhancement patterns and parameters correlate with the prognostic factors of tumor invasiveness to some extent. Hence, it is reasonable to assume that the different enhancement characteristics in CEUS may not only indicate microvascular distribution but also provide valuable information about tumor biologic potential and prognosis. The present study aimed to evaluate whether enhancement patterns and perfusion parameters correlate with microvascular distribution in breast cancer. In addition, the possible prognostic value of these parameters was assessed.

\section{Materials and methods}

Ethics statement. All patients who accepted SonoVue CEUS and who donated human breast tissues provided written informed consent prior to participation in the study. The protocols for collection and analysis of the samples were approved by the Institutional Review Board of the Tianjin Medical University Cancer Institute and Hospital (Tianjin, China), in accordance with the current revision of The Declaration of Helsinki.

Patient selection. From September 2011 to April 2013 in Tianjin Medical University Cancer Institute and Hospital, prospective analysis of 109 lesions in Breast Imaging Reporting and Data System (BI-RADS) classes 4-5 (7) was conducted by conventional breast ultrasound. Before core-needle biopsy, CEUS was performed. Following CEUS, punctures were performed on different enhancement regions in the same body position under the guidance of ultrasound. With the exception of 8 benign lesions, and 2 lesions in which biopsied tissue was too small to diagnose, the remaining malignant lesions $(n=99)$ confirmed by biopsy or surgical resection were enrolled. All patients were females, ranging in age from 19 to 93 years (mean age, 53.0 years).

Instruments and methods. All ultrasonographic examinations were performed using a Logiq E9 Ultrasound Machine (GE Healthcare Life Sciences, Chicago, IL, USA) with probe frequency of 6-15 MHz. The patient was required to lie down in a supine position with both breasts fully exposed, then the patient was examined under routine ultrasonographic mode for information including pathomorphology and color Doppler flow image. The most suspected lesion site was selected if the patient had multiple lesions. Once the routine mode exhibited the section of largest size or most abundant blood supply, it was switched to CEUS mode with probe frequency of $9 \mathrm{MHz}$ and SonoVue contrast agent was used (Bracco S.p.A., Milan, Italy; prepared according to the manufacturer's instructions). Contrast pulse sequencing was started and $2.5 \mathrm{ml}$ contrast agent was quickly administered using bolus injection via the cubital vein, followed by a $5 \mathrm{ml}$ normal saline flush. Dynamic perfusion of the lesion was observed in real time for $\geq 3 \mathrm{~min}$ after the injection. The whole process of dynamic CEUS was recorded and stored in the machine's hard drive and ultrasound workstation for further analysis.

Two ultrasonographers independently and blindly evaluated the CEUS images, and any disagreements were discussed until a consensus was reached. Enhancement patterns were defined based on the study objectives: 1) Homogeneous enhancement, all areas in the lesion site were homogeneously and diffusely enhanced with almost the same enhancement intensity; 2) peripheral enhancement, the periphery of the lesion site was enhanced without marked enhancement in the center of the lesion; or the periphery and center of lesion site were enhanced but the range and/or intensity of enhancement was more apparent in the periphery than the center; or 3) regional enhancement: The enhancement areas were distributed unevenly in the lesion site with different intensities; or a specific area of a lesion site presented homogeneous and diffuse enhancement.

Following CEUS, punctures were performed on differently enhanced regions in the same body position under guidance of ultrasound. A slice of tissue was taken for biopsy from the periphery and center of homogeneous and peripheral lesions, as well as from the different enhancement regions of a regional enhancement lesion. The selected cases consisted of $>2$ accurate puncture cores, intratumoral and peritumoral of breast cancer, to ensure that there were sufficient samples for a random selection of 5 different microscopic fields (5 within the tumor and 5 surrounding the tumor). The dynamic images stored on the machine's hard drive were examined at the corresponding puncture sites for time-intensity curves, in order to automatically obtain the perfusion parameters, including peak intensity (PI), ascending slope, time to peak, area under the time-intensity curve and wash-in time.

Histological types were defined according to the World Health Organization classification (8); histological grade was determined using the modified Bloom and Richardson grading system (9). The tumors were staged using the American Joint Committee on Cancer, 7th edition, TNM staging system (10).

Immunohistochemistry (IHC) and assessment of MVD. Tissues were fixed in $10 \%$ neutral-buffered formalin for $\sim 24 \mathrm{~h}$ at room temperature, then paraffin-embedded. Tissue sections $(5-\mu \mathrm{m})$ of intratumoral and peritumoral breast cancer from each case were selected. IHC for CD34 was performed according to standard procedures. In brief, $5-\mu \mathrm{m}$ tissue sections were sequentially dewaxed and rehydrated using xylene and graded alcohol washes. Antigen retrieval was performed at $121^{\circ} \mathrm{C}$ for $2 \mathrm{~min}$, using citrate buffer, $\mathrm{pH}$ 6.0. After serial blocking with hydrogen peroxide and normal goat serum (cat. no. ZLI-9056) for $10 \mathrm{~min}$ at room temperature, the sections were incubated with primary monoclonal antibody against CD34 (cat. no. ZA-0550; dilution 1:200) (both from Zhongshan Golden Bridge Biotechnology Co., Ltd., Beijing, China) for $16 \mathrm{~h}$ at $4^{\circ} \mathrm{C}$. The sections then were sequentially incubated with biotinylated goat anti-rabbit/mouse immunoglobulin (cat. no. SP-9000, working solution, undiluted; Zhongshan Golden Bridge Biotechnology Co., Ltd.) and peroxidase-conjugated streptavidin (Dako; Agilent Technologies, Inc., Santa Clara, CA, USA) for $20 \mathrm{~min}$ at $37^{\circ} \mathrm{C}$. The enzyme substrate was 3-3'-diaminobenzidine tetrahydrochloride. Incubation of 
Table I. Histopathological types of breast cancer among patients.

\begin{tabular}{lrr}
\hline Diagnosis & $\mathrm{n}$ & $\%$ \\
\hline DCIS & 3 & 3.0 \\
Invasive carcinoma & 96 & 97.0 \\
IDC-NOS & 83 & 83.8 \\
Mucinous carcinoma & 2 & 2.0 \\
Apocrine carcinoma & 2 & 2.0 \\
Neuroendocrine carcinoma & 1 & 1.0 \\
Mixed type carcinoma & 8 & 8.1 \\
\hline
\end{tabular}

Histological types were defined according to the World Health Organization classification. DCIS, ductal carcinoma in situ; IDC-NOS, invasive ductal carcinoma-not otherwise specified.

sections with phosphate-buffered saline served as the negative control.

MVD, as visualized by anti-CD34, was evaluated light microscopically. When CD34 IHC demonstrated cytoplasmic or membrane staining of endothelial cells or endothelial cell clusters that was clearly separated from adjacent clusters and background, with or without a lumen, this was recorded as an individual vessel. The fields containing the greatest numbers of microvessels (vascular hot-spots) were identified at low magnification (x10 objective lens). Then, microvessels within the above fields were counted at higher magnification (x40 objective lens). The total number of microvessel in each field was counted manually and confirmed by two investigators who were blinded to the clinicopathological characteristics and outcomes of patients. The average count from 5 fields within intratumoral and peritumoral breast cancer samples were recorded separately and used for statistical analysis.

Additional IHC for estrogen (ER; cat. no. ZM-0104; dilution 1:100), progesterone (PR; cat. no. ZM-0215; dilution 1:150) (both from Zymed; Thermo Fisher Scientific, Inc., Waltham, MA, USA), human epidermal growth factor receptor 2 (HER2, cat. no. c-erbB-2, dilution 1:400; Dako; Agilent Technologies, Inc.) and Ki-67 (cat. no. 081156; dilution 1:200; Zymed; Thermo Fisher Scientific, Inc.) of the tumors was performed on serial tissue sections of the core-needle biopsy using standard procedures. The immunohistochemical procedure is the same as aforementioned; the differences were that the sections were incubated with primary monoclonal antibody against for ER, PR, HER2 and Ki-67 for $2 \mathrm{~h}$ at $37^{\circ} \mathrm{C}$. The staining were analyzed using previously described criteria (11). For ER, PR and HER2, IHC was scored according to the American Society of Clinical Oncology/College of American Pathologists guidelines (12). Cases with a HER2 score of $\geq 2$ were further evaluated by fluorescence in situ hybridization using a Vysis kit (Abbott Laboratories, Abbott Park, IL, USA). Interpretation and scoring of Ki-67 staining was performed as described by Cheang et al (13).

Statistical analysis. SPSS 15.0 software (SPSS, Inc., Chicago, IL, USA) was used for statistical analyses. MVD ratio and CEUS-enhanced patterns were compared using the
Table II. Clinicopathological characteristics of patients with breast cancer.

\begin{tabular}{|c|c|}
\hline Characteristics & $\mathrm{n}$ \\
\hline $\mathrm{n}$ & 99 \\
\hline Age, years ${ }^{\mathrm{b}}$ & $53.0 \pm 10.8$ \\
\hline Tumor size, $\mathrm{mm}^{\mathrm{b}}$ & $22.7 \pm 9.4$ \\
\hline \multicolumn{2}{|c|}{ Histological grade, $\mathrm{n}(\%)^{\mathrm{a}}$} \\
\hline I & $8(9.6)$ \\
\hline II & $57(68.7)$ \\
\hline III & $18(21.7)$ \\
\hline \multicolumn{2}{|l|}{ TNM stage, n (\%) } \\
\hline Tis & $3(3.0)$ \\
\hline $\mathrm{T} 1$ & $42(42.4)$ \\
\hline $\mathrm{T} 2$ & $51(51.5)$ \\
\hline $\mathrm{T} 3$ & $3(3.0)$ \\
\hline \multicolumn{2}{|c|}{ Lymph node, n (\%) } \\
\hline Negative & $63(63.6)$ \\
\hline Positive & $36(36.4)$ \\
\hline \multicolumn{2}{|l|}{ ER status, n (\%) } \\
\hline Negative & $29(29.3)$ \\
\hline Positive & $70(70.7)$ \\
\hline \multicolumn{2}{|l|}{ PR status, n (\%) } \\
\hline Negative & $32(32.3)$ \\
\hline Positive & $67(67.7)$ \\
\hline \multicolumn{2}{|l|}{ HER2 status, n (\%) } \\
\hline Negative & $64(64.6)$ \\
\hline Positive & $35(35.4)$ \\
\hline \multicolumn{2}{|c|}{ Ki67 proliferative index, $\mathrm{n}(\%)$} \\
\hline Low & $25(25.3)$ \\
\hline High & $74(74.7)$ \\
\hline
\end{tabular}

${ }^{a}$ Only invasive ductal carcinoma-not otherwise specified type cases were included in histological grade analysis. ${ }^{b} \mathrm{Mean} \pm \mathrm{SD}$. TNM, tumor-node-metastasis; ER, estrogen; PR, progesterone; HER2, human epidermal growth factor receptor 2 .

Mann-Whitney U test. Correlations between MVD ratio and histological grade, TNM stage and CEUS parameters were analyzed using Spearman's rank correlation coefficient. The association between MVD ratio and histological characteristics of prognosis (including lymph node status, ER status, PR status, HER 2 status and Ki67 proliferative index) was analyzed using an independent-samples t-test. Two-sided $\mathrm{P}<0.05$ was considered to indicate a statistically significant difference.

\section{Results}

Characteristics of breast cancer patients. Histological types were defined according to the World Health Organization classification (8), including 83 cases $(83.8 \%$ ) of invasive ductal carcinoma-not otherwise specified type (IDC-NOS), 3 cases $(3.0 \%)$ of ductal carcinoma in situ, 2 cases $(2.0 \%)$ of apocrine carcinoma, 2 cases $(2.0 \%)$ of mucinous carcinoma, 1 case 
A
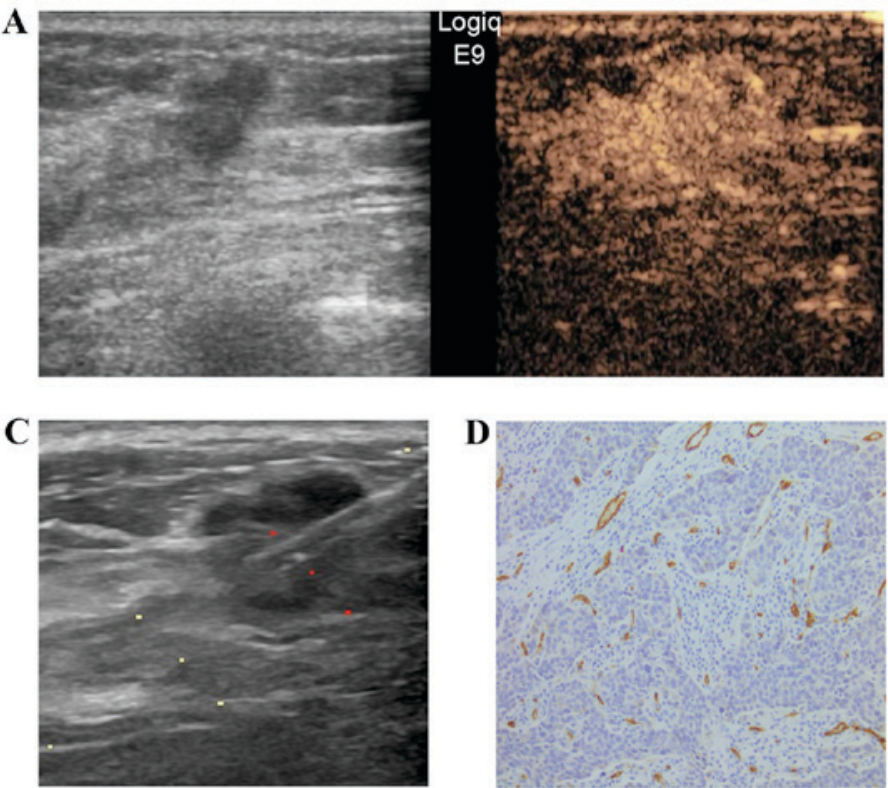

D

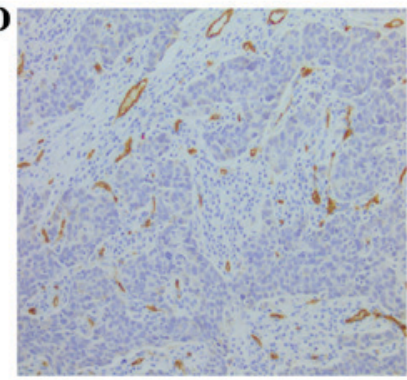

B

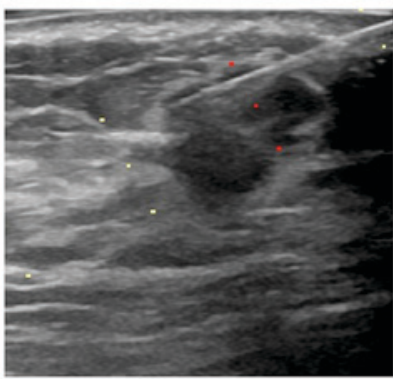

$\mathbf{E}$

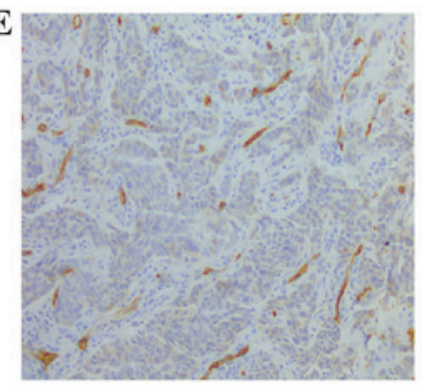

Figure 1. Homogenous enhancement. (A) Contrast-enhanced sonogram indicating a homogeneous enhancement mass. Core-needle biopsy from the (B) periphery and (C) center of the lesion. Immunohistochemical staining for CD34 indicates a similar distribution of microvessel density between the (D) periphery and (E) center of the lesion (original magnification, x200).
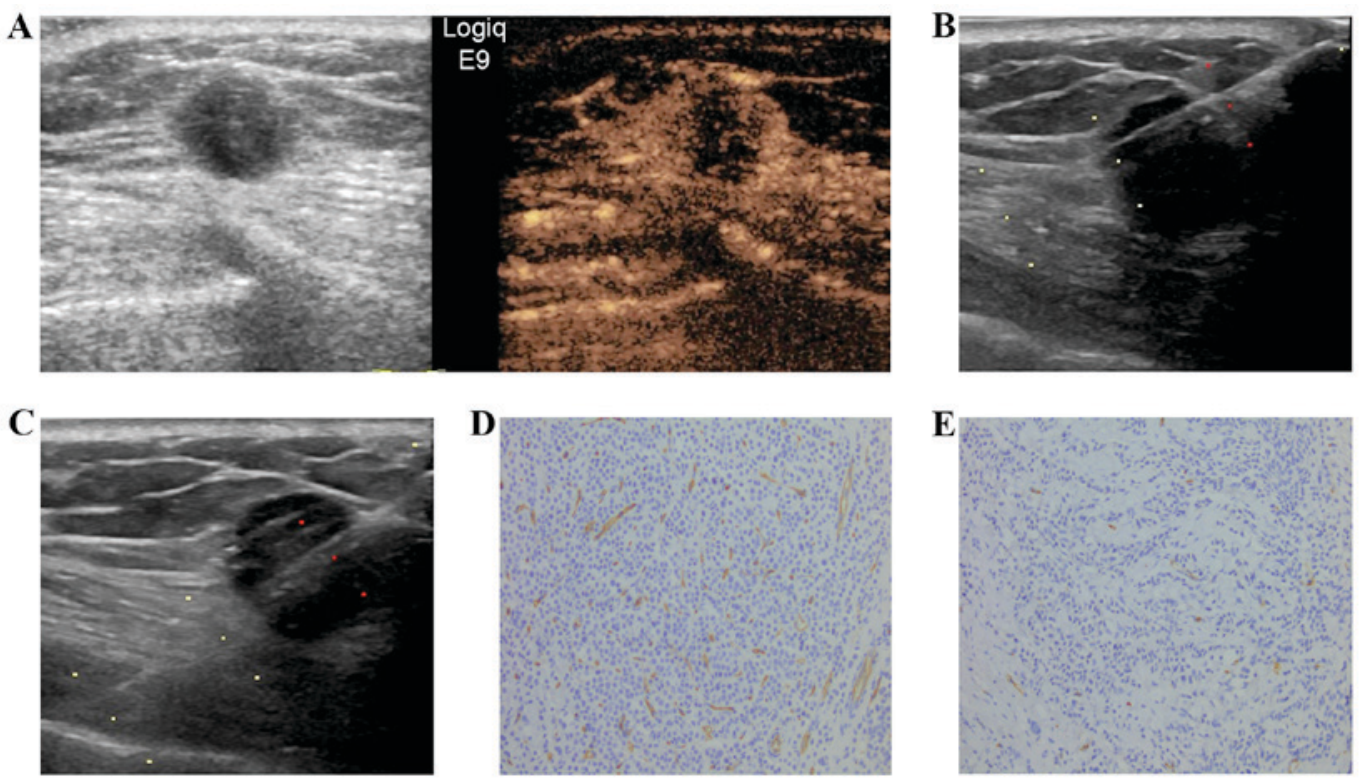

Figure 2. Peripheral enhancement. (A) Contrast-enhanced sonogram indicating a peripheral enhancement mass. Core-needle biopsy from the (B) periphery and (C) center of the lesion. Immunohistochemical staining for CD34 indicates (D) increased MVD in the periphery of the lesion, with (E) decreased MVD in the center of the lesion (original magnification, $\mathrm{x} 200$ ). MVD, microvessel density.

(1.0\%) of neuroendocrine carcinoma, and 8 cases $(8.1 \%)$ of mixed type carcinoma (Table I).

The characteristics of breast cancer patients are summarized in Table II. The average maximum tumor diameter was $23 \mathrm{~mm}$ (range, 6-75 mm). Histological grade was determined using the modified Bloom and Richardson grading system (9). Of the 83 IDC-NOS cases, 8 cases $(9.6 \%)$ were histological grade I, 57 cases $(68.7 \%)$ were histological grade II and 18 cases (21.7\%) were histological grade III. In all cases, the tumors were staged using the American Joint Committee on Cancer, 7th edition, TNM staging system (10) and included pTis $(n=3,3.0 \%)$, pT1 $(n=42,42.4 \%), p T 2(n=51,51.5 \%)$, pT3 $(n=3,3.0 \%)$. A total of $36(36.4 \%)$ patients exhibited lymph node metastasis, $70(70.7 \%)$ of the tumors were positive for ER, 67 (67.7\%) were positive for PR, 35 (35.4\%) exhibited HER2 overexpression and 74 (74.7\%) exhibited high Ki-67 proliferative index.

Correlation of MVD ratio with enhancement patterns and characteristics of breast cancer. In the present study, 44 (44.4\%) cases of primary breast cancer exhibited homogenous enhancement (Fig. 1), 43 (43.4\%) exhibited peripheral enhancement (Fig. 2) and 12 (12.1\%) exhibited regional enhancement (Fig. 3). Further analysis revealed differences 

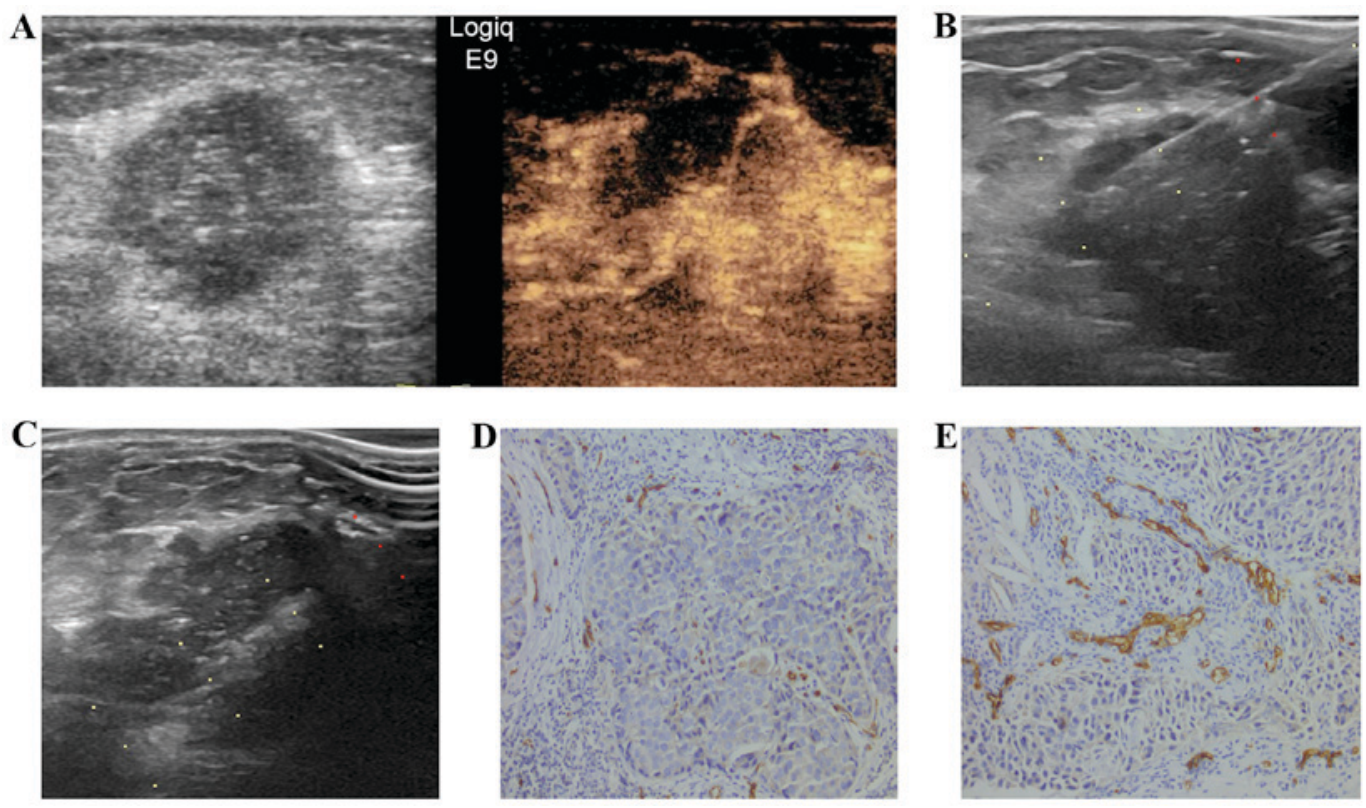

Figure 3. Regional enhancement. (A) Contrast-enhanced sonogram indicating a regional enhancement mass. Core-needle biopsy from an area of the lesion with (B) no enhancement and (C) obvious enhancement. Immunohistochemical staining for CD34 indicates decreased MVD in the (D) non-enhanced area of the lesion, with increased MVD in the (E) obviously enhanced area of the lesion (original magnification, x200). MVD, microvessel density.

Table III. Association between MVD ratio and contrast-enhanced ultrasound patterns in breast cancer.

\begin{tabular}{lccccccr}
\hline \multirow{2}{*}{ Enhancement pattern } & & \multicolumn{2}{l}{ MVD value, median (range) } & & MVD ratio of periphery to center, \\
\cline { 3 - 4 } & $\mathrm{n}$ & Periphery & Center & & Z & P-value \\
\hline Homogeneous enhancement & 44 & $69(12-157)$ & $59(11-155)$ & $1.04(0.90-2.13)$ & -3.604 & $<0.001$ \\
Peripheral enhancement & 43 & $66(14-330)$ & $53(8-223)$ & $1.23(1.00-4.50)$ & & \\
Regional enhancement $^{\mathrm{a}}$ & 12 & & & & & \\
\hline
\end{tabular}

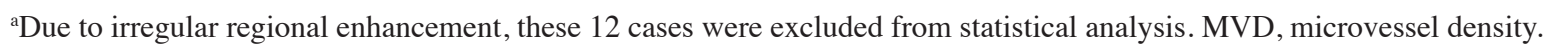

Table IV. Correlation between microvessel density ratio and contrast-enhanced ultrasound perfusion parameters in breast cancer ( $\mathrm{n}=87$ cases).

\begin{tabular}{lccccc}
\hline Statistic & Peak intensity ratio & Time to peak ratio & $\begin{array}{c}\text { Area under the time-intensity } \\
\text { curve ratio }\end{array}$ & $\begin{array}{c}\text { Ascending slope } \\
\text { ratio }\end{array}$ & $\begin{array}{c}\text { Wash-in time } \\
\text { ratio }\end{array}$ \\
\hline r-value & 0.267 & -0.061 & 0.159 & 0.271 & -0.050 \\
P-value & 0.017 & 0.591 & 0.163 & 0.016 & 0.660 \\
\hline
\end{tabular}

Analyzed using Spearman's rank-correlation test.

in MVD distribution between homogeneous enhancement, peripheral enhancement and regional enhancement. For homogeneous enhancement and peripheral enhancement, the respective medians of peripheral MVD were 69 (range, 12-157) and 66 (range, 14-330) and those of central MVD were 59 (range, 11-155) and 53 (range, 8-223). It was observed that the MVD periphery/center ratio in peripheral enhancement (median, 1.23; range, 1.00-4.50) was significantly higher compared with homogeneous enhancement of the lesions (median, 1.04; range, 0.90-2.13; $\mathrm{P}<0.001$ ). Furthermore, the enhancement characteristics and MVD values of corresponding biopsy sites were relatively similar in 12 regionally enhanced cases. These cases were not statistically analyzed or discussed further since there was no apparent association between enhancement region and microvascular distribution. The associations between CEUS enhancement patterns and MVD ratios are indicated in Table III.

Associations between MVD ratio and perfusion parameters of CEUS in breast cancer are presented in Table IV. PI and ascending slope ratios correlated with the corresponding 
Table V. Association between MVD ratio and histological characteristics of breast cancer.

\begin{tabular}{|c|c|c|c|c|c|}
\hline \multirow[b]{2}{*}{ Prognostic factors } & \multicolumn{2}{|c|}{ MVD value, median (range) } & \multirow{2}{*}{$\begin{array}{l}\text { MVD ratio periphery to } \\
\text { center, median (range) }\end{array}$} & \multirow{2}{*}{$\begin{array}{c}\text { R- or } \\
\text { T-value }\end{array}$} & \multirow[b]{2}{*}{ P-value } \\
\hline & Peripheral & Central & & & \\
\hline Histologic grade & & & & $0.364^{\mathrm{a}}$ & $0.003^{\mathrm{c}}$ \\
\hline $\mathrm{I}$ & $57(25-136)$ & $47(24-63)$ & $1.05(1.04-1.61)$ & & \\
\hline II & $67(45-88)$ & $59(33-74)$ & $1.09(1.02-1.29)$ & & \\
\hline III & $67(37-114)$ & $38(15-96)$ & $1.72(1.29-2.08)$ & & \\
\hline TNM stage & & & & $0.041^{\mathrm{a}}$ & $0.751^{\mathrm{c}}$ \\
\hline $\mathrm{T} 1$ & 59 (37-79) & $55(30-66)$ & $1.09(1.03-1.32)$ & & \\
\hline $\mathrm{T} 2$ & $70(64-92)$ & $60(36-75)$ & $1.07(1.02-1.41)$ & & \\
\hline $\mathrm{T} 3$ & $51(41-61)$ & $35(23-49)$ & $1.50(1.24-1.75)$ & & \\
\hline Lymph node status & & & & $-0.339^{b}$ & $0.735^{\mathrm{d}}$ \\
\hline Negative & $67(53-88)$ & $58(34-68)$ & $1.05(1.02-1.35)$ & & \\
\hline Positive & $70(42-95)$ & $56(31-78)$ & $1.17(1.03-1.48)$ & & \\
\hline ER status & & & & $0.052^{\mathrm{b}}$ & $0.958^{\mathrm{d}}$ \\
\hline Negative & $79(59-117)$ & $61(41-104)$ & $1.18(1.03-1.70)$ & & \\
\hline Positive & $67(42-82)$ & $53(31-69)$ & $1.10(1.02-1.33)$ & & \\
\hline PR status & & & & $-0.119^{b}$ & $0.906^{\mathrm{d}}$ \\
\hline Negative & $76(57-118)$ & $60(40-106)$ & $1.17(1.03-1.68)$ & & \\
\hline Positive & $66(42-82)$ & $51(29-68)$ & $1.10(1.02-1.33)$ & & \\
\hline HER2 status & & & & $1.725^{\mathrm{b}}$ & $0.088^{\mathrm{d}}$ \\
\hline Negative & $67(48-94)$ & $53(32-67)$ & $1.16(1.03-1.62)$ & & \\
\hline Positive & $73(43-92)$ & $65(34-90)$ & $1.06(1.02-1.31)$ & & \\
\hline Ki67 proliferative index & & & & $1.308^{\mathrm{b}}$ & $0.195^{\mathrm{d}}$ \\
\hline Low & $67(34-77)$ & $29(21-57)$ & $1.13(1.01-1.61)$ & & \\
\hline High & $67(52-81)$ & $58(38-68)$ & $1.10(1.02-1.44)$ & & \\
\hline
\end{tabular}

${ }^{a}$ r-value; ${ }^{b}$ t-value; ${ }^{c}$ analyzed using Spearman's rank-correlation test; ${ }^{d}$ analyzed using independent-samples t-test. MVD, microvessel density; TNM, tumor-node-metastasis; ER, estrogen; PR, progesterone; HER2, human epidermal growth factor receptor 2.

MVD ratios $(\mathrm{P}=0.017$ and $\mathrm{P}=0.016$, respectively). However, no significant correlation was observed between MVD ratio and the other parameters, including wash-in time, time to peak and area under the time-intensity curve.

Association between MVD ratio and histological characteristics of breast cancer. Associations between MVD ratio and histological characteristics of breast cancer are presented in Table V. MVD ratio was positively correlated with histological grade $(\mathrm{P}=0.003)$. However, no significant associations were observed between MVD ratio and other prognostic factors, including TNM stage, lymph node status, expression of ER and PR, Ki67 proliferative index and HER2 overexpression.

\section{Discussion}

Multiple factors correlate with different enhancement presentations in images of breast cancer, among which MVD and microvascular distribution play the most important roles in the enhancement patterns of tumors (14). CEUS of breast is a non-invasive method for evaluating the degree of tumor vascularization, which uses blood pool contrast agents that cannot easily pass into intercellular spaces. Thus, it is appropriate for the evaluation of microcirculation perfusion (15). A previous study demonstrated that CEUS patterns and parameters correlate well with MVD and microvascular distribution in lesions, which have focused on the CEUS characteristics of high- and low-MVD lesions (5). In the present study, different CEUS-enhanced regions of breast cancer were used for biopsy specimens, aiming at a more accurate evaluation of the distribution differences of microvascular regions in breast cancer and the possible prognoses indicated by these differences.

Different CEUS enhancement patterns at different regions of breast cancer are primarily attributed to blood perfusion disparities. Pathological examinations reveal that blood vessel-rich regions are located at the peritumoral areas of breast cancer (14). The majority of peripheries have tortuous and dilated large vessels, while intratumoral vessels of breast cancer are often naive, narrow and obstructed (16,17). Previous studies have demonstrated that peripheral enhancement of magnetic resonance imaging in breast cancer is associated with a higher MVD of the peripheral region as compared with the central region $(14,18)$. However, to the best of our knowledge, no reports have focused on the correlation between different CEUS enhancement patterns of breast cancer and microvascular distribution. In the present study, it was observed that MVD of the periphery was 
higher compared with MVD of the center in breast cancer. A higher peripheral/central MVD ratio was observed in peripherally enhanced lesions compared with homogeneously enhanced lesions, indicating a higher extent of vascularization in peripherally enhanced lesions. Furthermore, the CEUS enhancement characteristics and MVD values of corresponding puncturing sites were relatively similar in 12 regionally enhanced cases. These cases were not statistically analyzed or discussed further since there was no apparent association between enhancement region and microvascular distribution.

Quantitative analysis with CEUS provides an objective and reproducible method for the evaluation of the degree of vascularization. The results of the present study reveal that peripheral/central MVD ratio is associated with PI ratio and ascending slope ratio. PI reflects the quantity of contrast agent microbubbles in the vascular bed of the lesion; while ascending slope reflects the early flow quantity and velocity during contrast agent perfusion. The two parameters are associated with the degree of vascularization.

Biological prognostic factors of tumors (including histological grade, TNM stage, MVD, vascular endothelial growth factor, Ki-67, HER2, ER and PR) reflect the biological behaviors and prognosis of breast cancer to some extent (19). Furthermore, MVD is regarded as an independent prognostic factor of breast cancer and is correlated with histological grade and proliferative activity to a certain degree $(18,20,21)$. The current results indicated a significant correlation between the peripheral/central MVD ratio and histological grade of breast cancer $(\mathrm{P}=0.003)$, consistent with the findings of Fridman et al (21). A higher peripheral/central MVD ratio was observed in peripherally enhanced lesions compared with homogeneously enhanced lesions, which may be associated with poor prognosis.

While advantages of CEUS application in breast cancer have been demonstrated, limitations also exist in the present study. First, only a specific section from a lesion site can be selected by CEUS for the evaluation of enhancement patterns and parameters, and it is impossible to wholly observe the lesion. Thus, the histopathological characteristics obtained from core-needle biopsy cannot represent the status of the whole tumor. Second, certain subjective and procedure errors are unavoidable. In conclusion, the enhancement patterns and parameters of CEUS may not only reflect the microvessel distribution but also indirectly indicate the histological grade of breast cancer. As a non-invasive examination technique, CEUS of breast cancer can objectively reflect the basic pathological characteristics of blood supply to the breast tumor, and is helpful in evaluating the biological behaviors and prognosis of breast tumor.

\section{Acknowledgements}

This study was supported by the Program of Science and Technology Project of Anticancer, Tianjin, China (grant no. 12ZCDZSY16000) and the Medical University of Tianjin Cancer Hospital Doctor Startup Foundation (grant no. 1314) awarded to Yaqing Li.

\section{References}

1. Fan F, Schimming A, Jaeger D and Podar K: Targeting the tumor microenvironment: Focus on angiogenesis. J Oncol 2012: 281261, 2012 .
2. Hida K, Kawamoto T, Ohga N, Akiyama K, Hida Y and Shindoh M: Altered angiogenesis in the tumor microenvironment. Pathol Int 61: 630-637, 2011.

3. Zhao H, Xu R, Ouyang Q, Chen L, Dong B and Huihua Y: Contrast-enhanced ultrasound is helpful in the differentiation of malignant and benign breast lesions. Eur J Radiol 73: 288-293, 2010.

4. Vallone P,D'Angelo R, Filice S, Petrosino T, Rinaldo M, De Chiara A and Gallipoli A: Color-doppler using contrast medium in evaluating the response to neoadjuvant treatment in patients with locally advanced breast carcinoma. Anticancer Res 25: 595-599, 2005.

5. Du J, Li FH, Fang H, Xia JG and Zhu CX: Correlation of real-time gray scale contrast-enhanced ultrasonography with microvessel density and vascular endothelial growth factor expression for assessment of angiogenesis in breast lesions. J Ultrasound Med 27: 821-831, 2008.

6. Wan CF, Du J, Fang H, Li FH, Zhu JS and Liu Q: Enhancement patterns and parameters of breast cancers at contrast-enhanced US: Correlation with prognostic factors. Radiology 262: 450-459, 2012.

7. American College of Radiology (ACR): Breast Imaging Reporting and Data System Atlas (BI-RADS Athas). 4th edition. ACR, Reston, VA, 2013.

8. Lakhani SR, Ellis IO, Schnitt SJ, Tan PH and van de Vijver MJ (eds): WHO Classification of Tumours of the Breast. Vol 4. 4th edition. IARC Press, 2012.

9. Elston CW and Ellis IO: Pathological prognostic factors in breast cancer. I. The value of histological grade in breast cancer: Experience from a large study with long-term follow-up. Histopathology 41: 154-151, 2002.

10. AJCC Cancer Staging Handbook: From the AJCC Cancer Staging Manual. Edge S, Byrd DR, Compton CC, Fritz AG, Greene F and Trotti A (eds). 7th edition. Springer-Verlag, NY, 2010.

11. Liu F, Lang R, Zhao J, Zhang X, Pringle GA, Fan Y, Yin D, Gu F, Yao $\mathrm{Z}$ and $\mathrm{Fu} \mathrm{L}: \mathrm{CD}^{+}$cytotoxic $\mathrm{T}$ cell and $\mathrm{FOXP}^{+}$regulatory $\mathrm{T}$ cell infiltration in relation to breast cancer survival and molecular subtypes. Breast Cancer Res Treat 130: 645-655, 2011.

12. Hammond ME, Hayes DF, Dowsett M, Allred DC, Hagerty KL, Badve S, Fitzgibbons PL, Francis G, Goldstein NS, Hayes M, et al: American Society of Clinical Oncology/College of American Pathologists guideline recommendations for immunohistochemical testing of estrogen and progesterone receptors in breast cancer (unabridged version). Arch Pathol Lab Med 134: e48-e72, 2010.

13. Cheang MC, Chia SK, Voduc D, Gao D, Leung S, Snider J, Watson M, Davies S, Bernard PS, Parker JS, et al: Ki67 index, HER2 status, and prognosis of patients with luminal B breast cancer. J Natl Cancer Inst 101: 736-750, 2009.

14. Buadu LD, Murakami J, Murayama S, Hashiguchi N, Sakai S, Masuda K, Toyoshima S, Kuroki S and Ohno S: Breast lesions: Correlation of contrast medium enhancement patterns on MR images with histopathologic findings and tumor angiogenesis. Radiology 200: 639-649, 1996.

15. Liu H, Jiang YX, Liu JB, Zhu QL and Sun Q: Evaluation of breast lesions with contrast-enhanced ultrasound using the microvascular imaging technique: Initial observations. Breast 17: 532-539, 2008.

16. Nagy JA, Chang SH, Shih SC, Dvorak AM and Dvorak HF: Heterogeneity of the tumor vasculature. Semin Thromb Hemost 36: 321-331, 2010.

17. Jackson A, O'Connor JP, Parker GJ and Jayson GC: Imaging tumor vascular heterogeneity and angiogenesis using dynamic contrast-enhanced magnetic resonance imaging. Clin Cancer Res 13: 3449-3459, 2007.

18. Teifke A, Behr O, Schmidt M, Victor A, Vomweg TW, Thelen M and Lehr HA: Dynamic MR imaging of breast lesions: Correlation with microvessel distribution pattern and histologic characteristics of prognosis. Radiology 239: 351-360, 2006.

19. Kushlinskii NE, Orinovskii MB, Gurevich LE, Kazantseva IA, Talaeva ShZh, Shirokii VP, Ermilova VD, Dvorova EK and Ozherelev AS: Expression of biomolecular markers (Ki-67, PCNA, Bcl-2, BAX, BclX, VEGF) in breast tumors. Bull Exp Biol Med 137: 182-185, 2004.

20. Weidner N, Folkman J, Pozza F, Bevilacqua P, Allred EN, Moore DH, Meli S and Gasparini G: Tumor angiogenesis: A new significant and independent prognostic indicator in early-stage breast carcinoma. J Natl Cancer Inst 84: 1875-1887, 1992.

21. Fridman V, Humblet C, Bonjean K and Boniver J: Assessment of tumor angiogenesis in invasive breast carcinomas: Absence of correlation with prognosis and pathological factors. Virchows Arch 437: 611-617, 2000. 